\title{
Distribution of type IV collagen immunoreactivity to assess questionable early stromal invasion
}

\author{
C J R Stewart, A M McNicol
}

\begin{abstract}
Aims: To determine if the immunocytochemical delineation of subepithelial basement membrane can be used in the assessment of questionable early invasive cervical carcinoma.

Methods: The distribution of immunoreactive type IV collagen was examined in 15 cervical biopsy specimens in which the reporting pathologist had specifically described difficulty in assessing or excluding early invasion of subepithelial stroma associated with cervical intraepithelial neoplasia (CIN). The results were compared with those from biopsy specimens showing CIN III $(\mathbf{N}=8)$, carcinoma with definite early stromal infiltration (FIGO stage 1a1) $(n=6)$, and more advanced invasive squamous tumours (FIGO stages $1 \mathrm{a} 2$ to 3$)(n=8$ ). In all cases the immunocytochemical findings were assessed in relation to serial sections stained with haematoxylin and eosin.
\end{abstract}

Results: Six of the 15 diagnostically problematic biopsy specimens were considered, on review, to show early infiltration of subepithelial tissue and putative invasive foci were consistently absent in basement membrane. A similar pattern was observed in the "definite" early invasive cases. Eight problematic biopsy specimens were considered to show only in situ neoplasia; five of these had intact though occasionally attenuated basement membrane, three showed focal type IV collagen defects. In the remainfurther assessment. Basement memeight cases of CIN III, while three of eight squamous carcinomas showed partial retention of type IV collagen immunoreactivity around invasive tumour cell nests.

Department of Pathology, Royal Infirmary, Glasgow G4 OSF

C J R Stewart

A M McNicol

Correspondence to Dr C J R Stewart

Accepted for publication 31 July 1991

Conclusions: Defects in subepithelial basement membrane occur in in situ and invasive neoplasia in the uterine cervix. Immunocytochemical staining for type IV collagen is of limited diagnostic value in the assessment of biopsy specimens with questionable early stromal ing case biopsy trauma precluded brane defects were identified in five of

The FIGO classification of microinvasive squamous carcinoma of the uterine cervix was refined in 1985 and a new subgroup of stage 1a disease (stage 1al) that incorporates tumours showing only early stromal invasion was defined. ${ }^{1}$ The recognition of early stromal invasion in histological sections, however, is not always straightforward, particularly in small, possibly traumatised or tangentially orientated punch biopsy specimens, or in the presence of a substantial stromal inflammatory infiltrate. ${ }^{2}$ As penetration of the subepithelial basement membrane is generally considered to be the definitive diagnostic feature of early stromal invasion, ${ }^{3}$ considerable interest has been shown in the immunocytochemical demonstration of basement membrane constituents such as laminin and type IV collagen in tumours from various sites. The initial view that basement membrane is uniformly intact in benign conditions and focally defective in malignancy ${ }^{4}$ cannot, however, be sustained. ${ }^{5}$ Using immunocytochemical techniques, defects in basement membranes have been shown in normal tissues as well as in benign pathological processes, ${ }^{67}$ and conversely, partial persistence or resynthesis of basement membrane has been described in invasive malignancy including nodal metastases. ${ }^{8-10}$

Despite these findings it has been suggested that immunocytochemical demonstration of basement membrane components may be useful in assessing questionable early invasive disease in cervical biopsy specimens. ${ }^{7911}$ Previous studies, however, have not actually tested this hypothesis in problematic diagnostic cases. We therefore examined type IV collagen immunoreactivity in a series of cervical biopsy specimens in which the reporting pathologist had specifically commented on the difficulty of excluding stromal invasion using conventionally stained (haematoxylin and eosin) sections. For comparison, cases showing cervical intraepithelial neoplasia, definite early stromal invasion, or frank invasive carcinoma were also assessed. invasion.
TISSUES

Diagnostic punch or cone cervical biopsy specimens were taken from patients who had had an abnormal cervical smear. The

\section{Methods}


specimens were fixed in Bouin's solution or $10 \%$ buffered formalin and processed to paraffin wax. Serial $4 \mu \mathrm{m}$ sections were stained with haematoxylin and eosin and type IV collagen immunocytochemistry. Four groups of diagnostic cases were examined.

\section{Group A: cervical intraepithelial neoplasia, grade III (CIN III)}

Eight biopsy specimens were assessed and graded according to the criteria of Buckley, Butler, and Fox. ${ }^{12}$ Neoplastic epithelium had extended into endocervical crypts in four cases but none showed evidence of stromal infiltration.

\section{Group B: early stromal invasion}

Invasion of the stroma was diagnosed using the criteria outline by Sedlis et al. ${ }^{2}$ based on changes in tumour differentiation and stromal response. In all six cases the invasive cells were continuous with and arising from CIN ("budding invasion"), or present as separated single cells close to the basement membrane of in situ disease. The criteria for FIGO stage lal cervical carcinoma had therefore been fulfilled.

\section{Group C: invasive squamous carcinoma}

Sections from stages $1 \mathrm{a} 2(\mathbf{n}=2), \mathbf{l b}(\mathbf{n}=3)$, $2(n=2)$, and $3(n=1)$ cervical squamous carcinomas were assessed. The tumours were subjectively graded as well differentiated $(n=2)$, moderately differentiated $(n=3)$, or poorly differentiated $(\mathrm{n}=3)$ according to standard criteria. Mucin stains were negative in all cases.

\section{Group D: problematic diagnostic cases}

A series of diagnostically difficult cases was obtained by reviewing the histopathology reports of tumour coded cervical biopsy specimens over a six year period to 1990 . In each specimen the reporting pathologist had

Figure 1 CIN III associated with a stromal inflammatory infiltrate. There are focal defects in type IV collagen immunostaining (arrows) but no morphological features of invasive carcinoma. $(A)$

haematoxylin and eosin (B) immunoperoxidase (no counterstain).

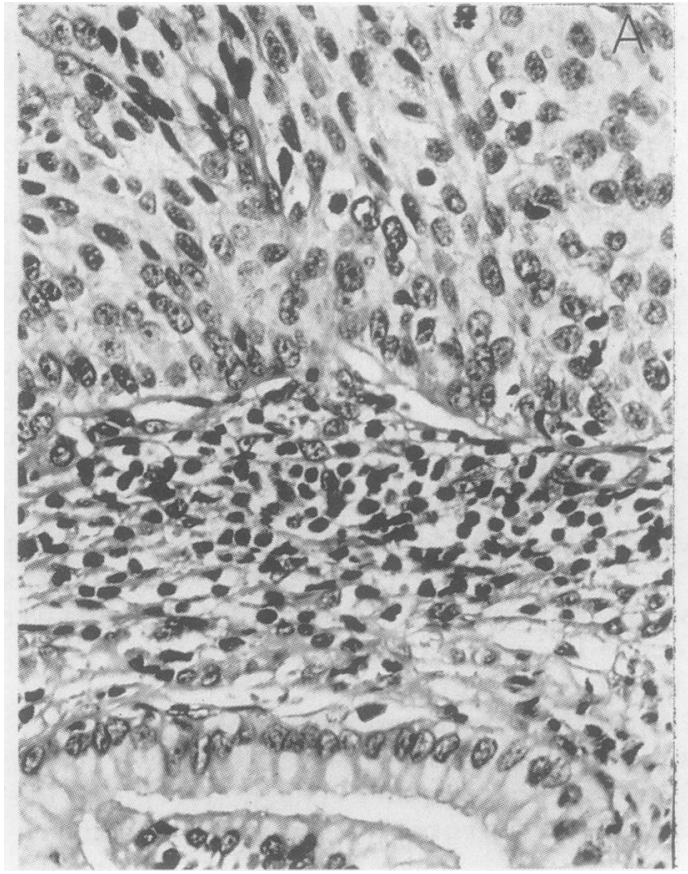

specifically reported difficulty in assessing or confidently excluding early stromal invasion associated with CIN. Difficulty in excluding invasion was usually considered to be related to a heavy stromal inflammatory response, limited diagnostic material, or to biopsy trauma. Routinely stained sections from each biopsy specimen were reassessed and where possible, a putative conclusion on the presence or absence. of stromal invasion was reached.

\section{IMMUNOCYTOCHEMISTRY}

Sections $(4 \mu \mathrm{m})$ were stained using a standard indirect immunoperoxidase technique with a primary mouse monoclonal antibody to type IV collagen (Serotec) incubated for two hours at a dilution of 1 in 250 . The secondary antibody was a goat anti-mouse/horseradish peroxidase conjugate (Dako) incubated for 30 minutes at a dilution of 1 in 50. Diaminobenzidine was used as a chromogen in all cases. Optimal staining of basement membrane was obtained by pretreating sections with protease XXVII (Sigma) digestion for 20 minutes (biopsy specimens fixed in Bouin's solution) or 60 minutes (formalin fixed) at $37^{\circ} \mathrm{C}$. In control sections the primary antiserum was substituted with normal mouse serum.

Type IV collagen immunoreactivity was assessed in relation to corresponding haematoxylin and eosin stained serial sections.

\section{Results}

In all biopsy specimens examined type IV collagen immunoreactivity gave strong, well defined staining of the basement membranes of normal squamous epithelium and of vessel walls. Basement membranes of endocervical crypts were generally less intensely stained and sometimes appeared thicker and less well defined due to partial tangential cutting. In

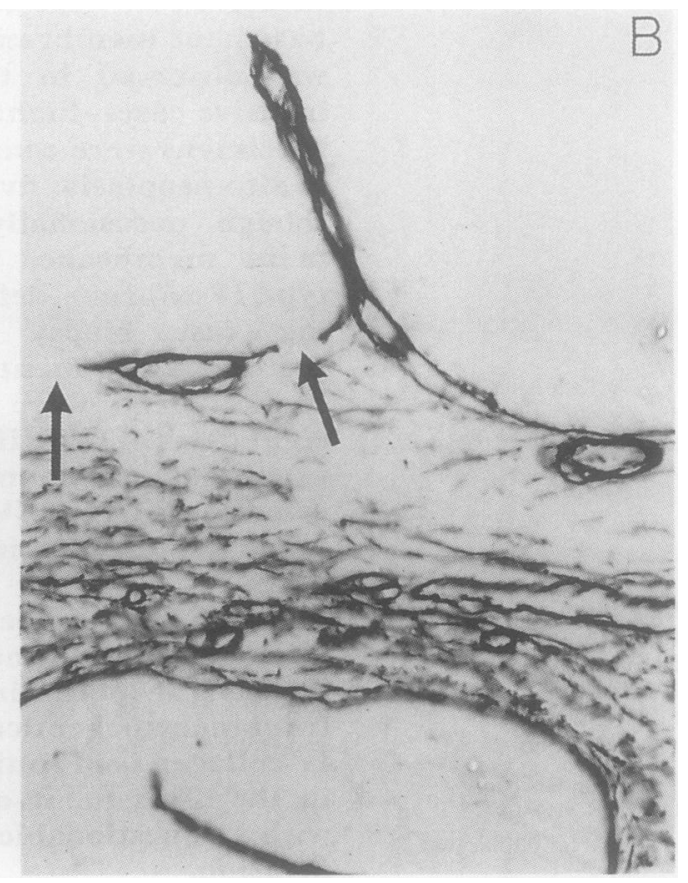


Figure 2 Early stromal invasion in continuity with CIN. Basement membrane is absent around the invasive nest but retained in adjacent vascular structures. ( $A$ ) haematoxylin and eosin; (B) immunoperoxidase (no counterstain).
Figure 3 Invasive squamous carcinoma. The larger tumour cell aggregate (top right) shows partial retention of type IV collagen, whereas a small group of infiltrating cells (long arrow) has no surrounding basement membrane. A focus of intravascular invasion is also present (short arrow); the vascular basement membrane is intact. $(A)$ haematoxylin and eosin; $(B)$ immunoperoxidase.

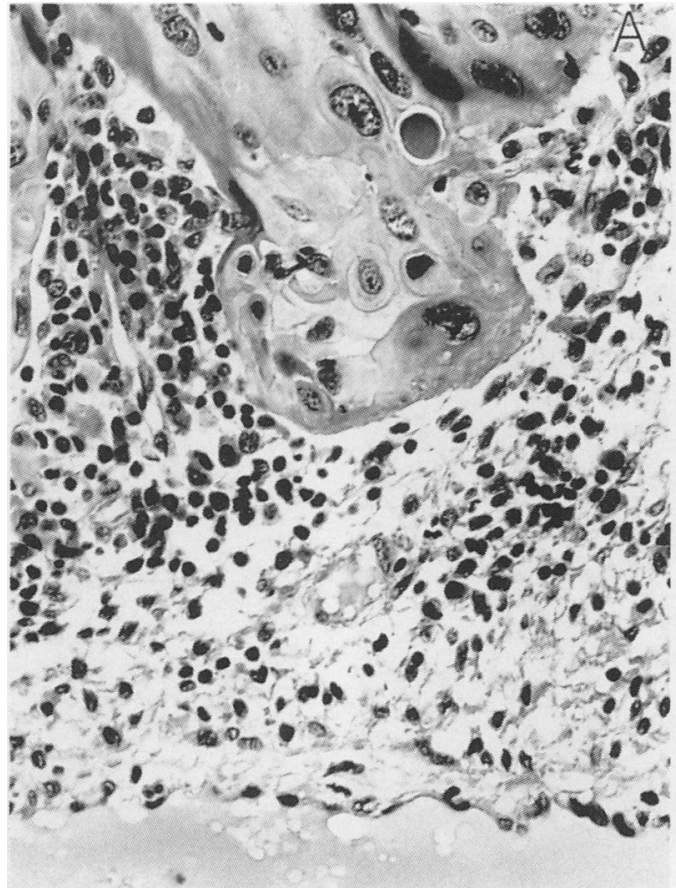

most specimens focal disruption of type IV collagen staining due to traumatic artefact was evident. In most cases this was of no diagnostic concern as the cause of the basement membrane loss was apparent on corresponding haematoxylin and eosin stained sections.

Group $A$ (CIN III)

In CIN III the basement membrane was generally intact and continuous, even in areas associated with a stromal inflammatory infiltrate. Multiple small defects that were apparently unrelated to trauma were observed, however, in five cases (fig 1). Re-examination of the corresponding stained consecutive serial sections in each biopsy specimen showed none of the recognised features of stromal invasion in these areas.

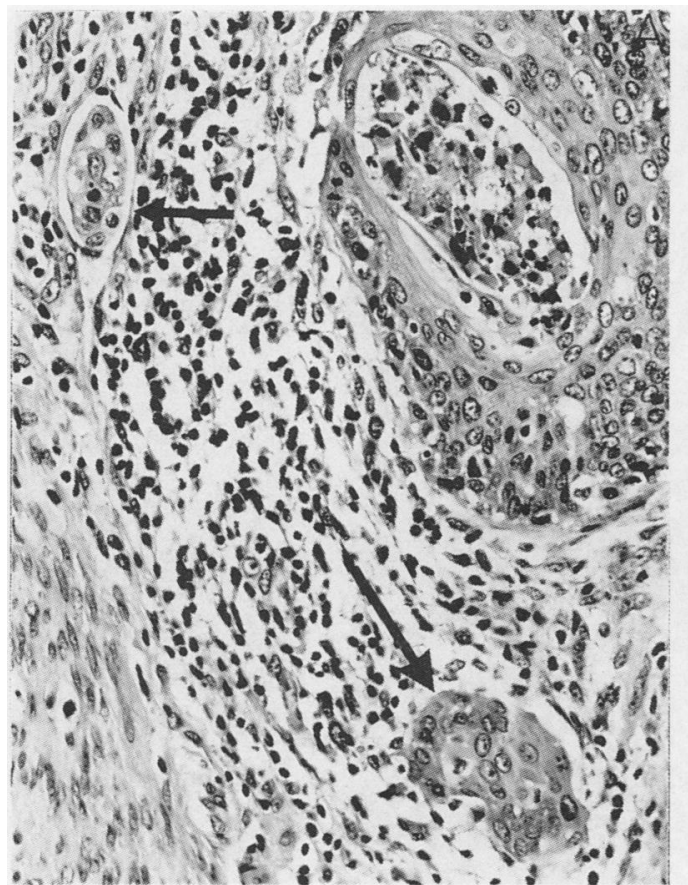

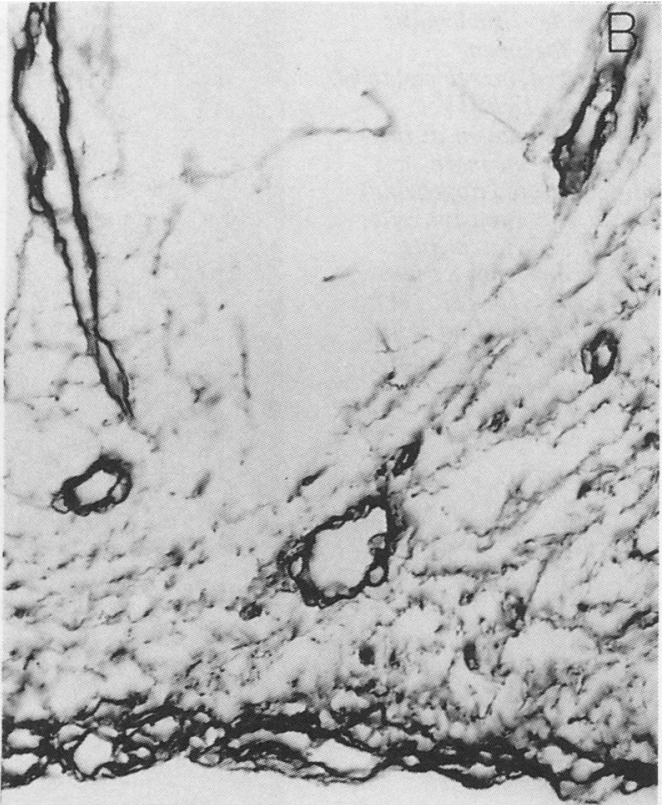

Group B (early stromal invasion)

In all cases type IV collagen immunoreactivity was absent around areas showing budding invasion contiguous with CIN (fig 2), and in infiltrating single malignant cells in stroma. Conventional histological assessment showed that staining correlated closely with the extent of invasion. By contrast, the basement membrane in adjacent areas of intraepithelial neoplasia was generally intact, although loss of imunoreactivity in supposedly in situ areas was also observed in two cases.

\section{Group C (invasive squamous carcinoma)}

Variations in basement membrane pattern were observed. In four out of eight cases there was no clear type IV collagen staining around infiltrating tumour cells. This was not easily

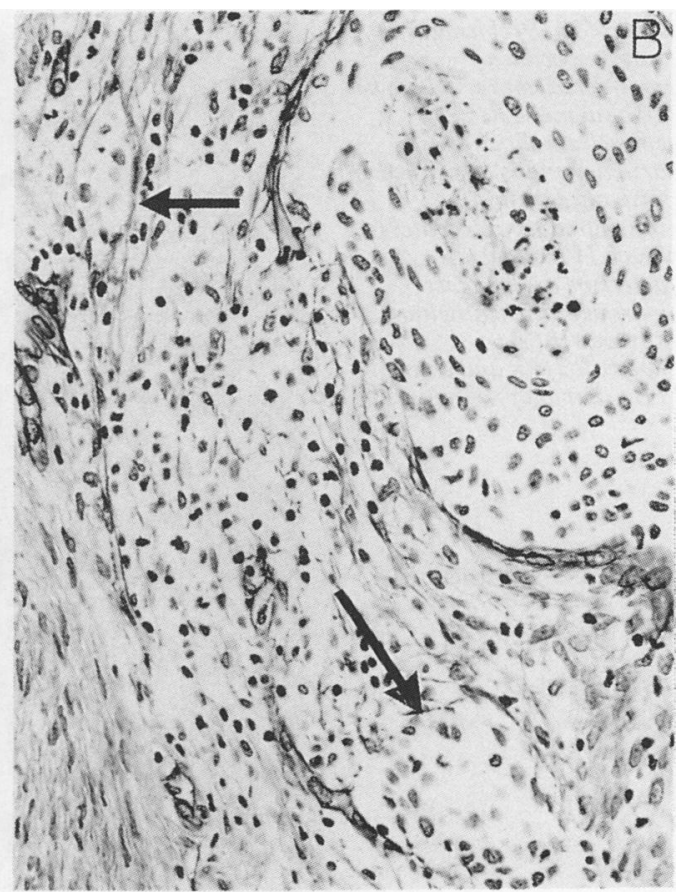


Figure 4 Problematic biopsy specimen considered, on review, to be invasive. Type IV collagen is absent at the margin of the more differentiated appearing, putatively invasive cells (arrows). Basement membrane is intact in adjacent CIN III. ( $A$ ) haematoxylin and eosin (B) immunoperoxidase (no counterstain).

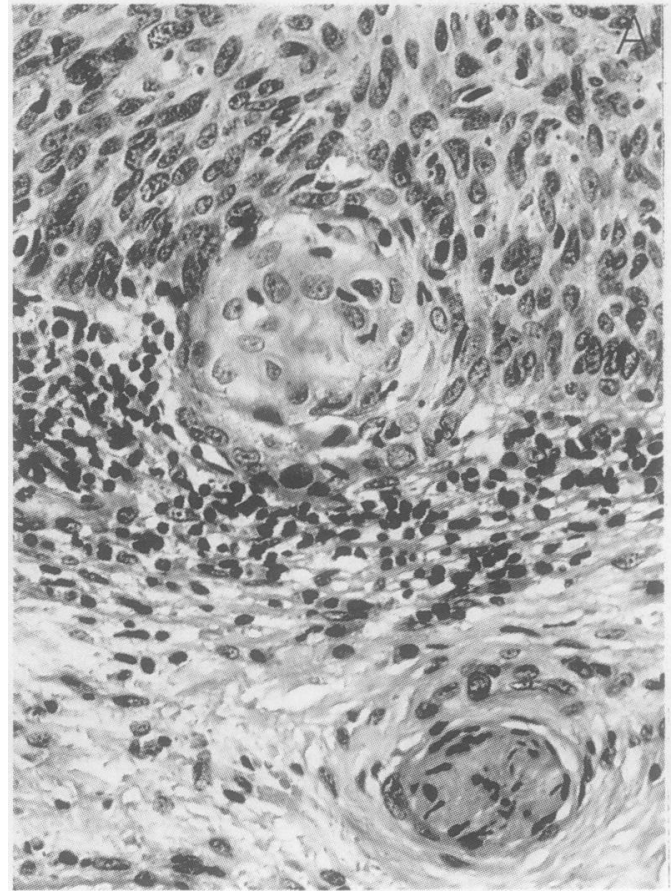

assessed in all areas, however, as compression of stromal elements, particularly vascular channels, at the margin of tumour aggregates gave the impression of a surrounding intact basement membrane. Tumour permeation of vascular channels created similar difficulties in immunocytochemical sections, but these were usually resolved when the corresponding stained sections were examined. The remaining four invasive tumours exhibited partial type IV collagen imunoreactivity around larger tumour nests, although staining was consistently absent in related to individually infiltrating cells (fig 3 ). There was no obvious correlation between tumour stage or grade and basement membrane pattern in the cases examined.

Figure 5 Problematic biopsy specimen considered, on review, to be CIN alone. The epithelium shows crush artefact and is associated with a substantial inflammatory infiltrate. Type IV collagen is defective at the lower margin of the epithelium (arrows). ( $A$ )

haematoxylin and eosin

(B) immunoperoxidase.

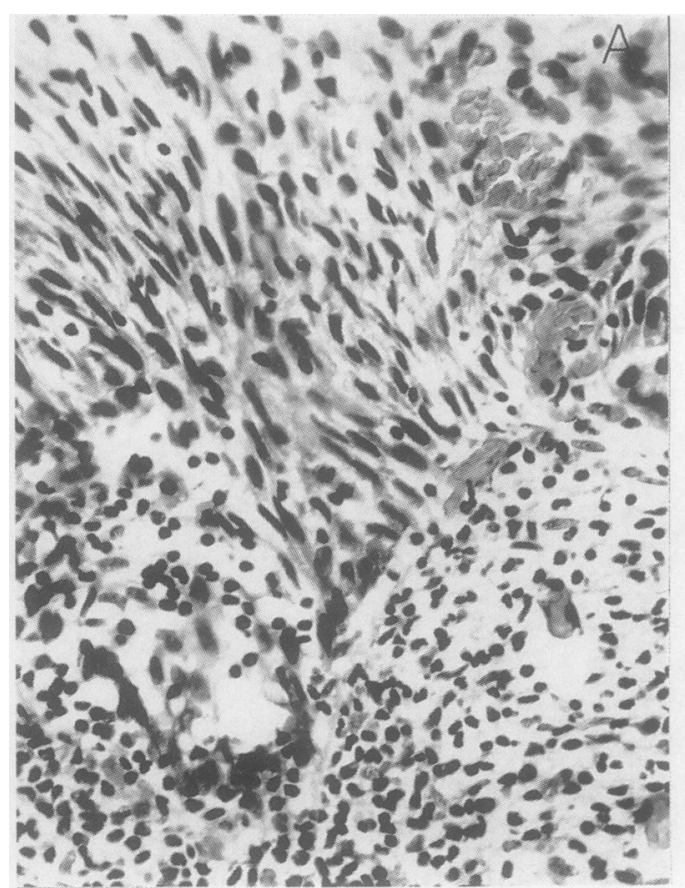

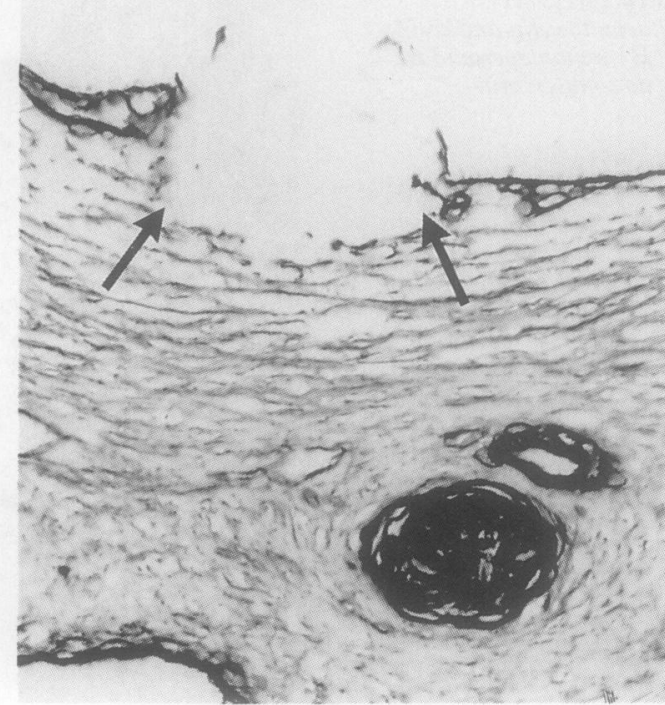

Group D (problem cases)

Following a review of the conventionally stained sections, six out of the 15 biopsy specimens were considered to show microinvasive disease. Eight cases were thought to represent intraepithelial neoplasia and biopsy trauma precluded further assessment of the one remaining biopsy specimen.

In the six biopsy specimens that had been reassessed as showing early stromal invasion basement membrane was consistently absent around putative invasive foci (fig 4). The eight specimens which were considered to show only in situ neoplasia were usually associated with an inflammatory infiltrate which disrupted the epithelial/stromal interface. Immunocytochemistry showed the basement membrane to

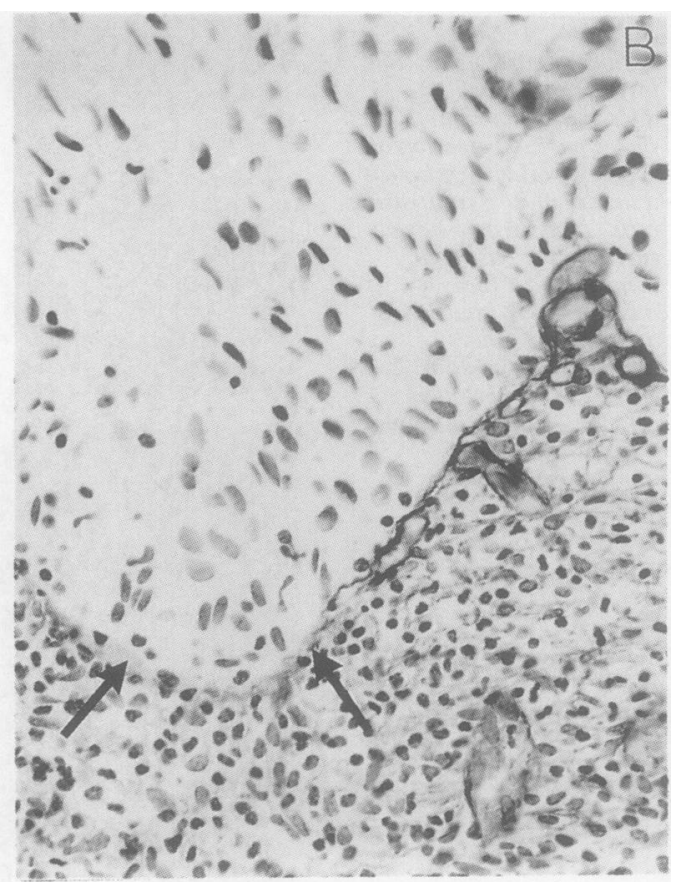


Figure 6 Problematic biopsy specimen

considered, on review, to be CIN alone. Nests of cells show increased cytoplasmic differentiation suggestive of budding invasion. Type IV collagen is intact but focally attenuated (arrow). ( $A$ ) haematoxylin and eosin (B) immunoperoxidase.

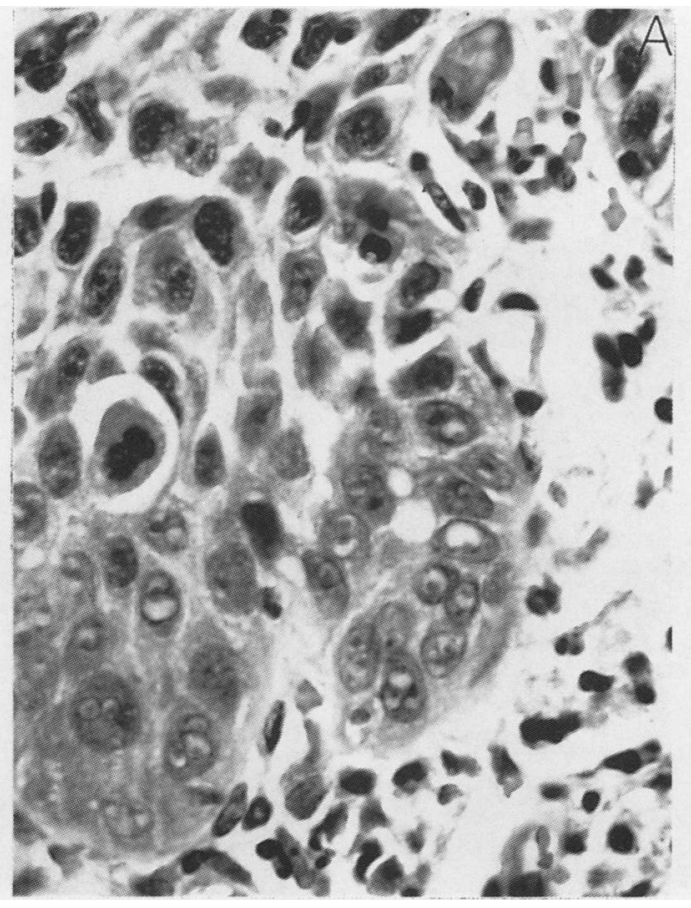

be focally defective in three cases (fig 5), and intact, though often less distinctly stained, in five (figs 6 and 7). Decidualisation of stroma was evident in one biopsy specimen: type IV collagen surrounded individual decidualised cells (fig 8). Focal breaks in basement membrane were also apparent in adjacent CIN; these were unrelated to the areas presenting diagnostic difficulties.

\section{Discussion}

The implementaton of the cervical screening programme has led to a sharp increase in the number of diagnostic cervical biopsy specimens examined in most histopathology departments. The detection of asymptomatic cervical lesions has created a unique opportun-

Figure 7 Problematic biopsy specimen considered, on review, to be CIN alone. CIN III in an endocervical crypt at top and right, an apparently discontinuous focus of epithelium with surrounding inflammatory infiltrate, and indistinct epithelial/stromal interface arrow). Basement membrane staining is intact in both areas. $(A)$ haematoxylin and eosin, (B) immunoperoxidase.

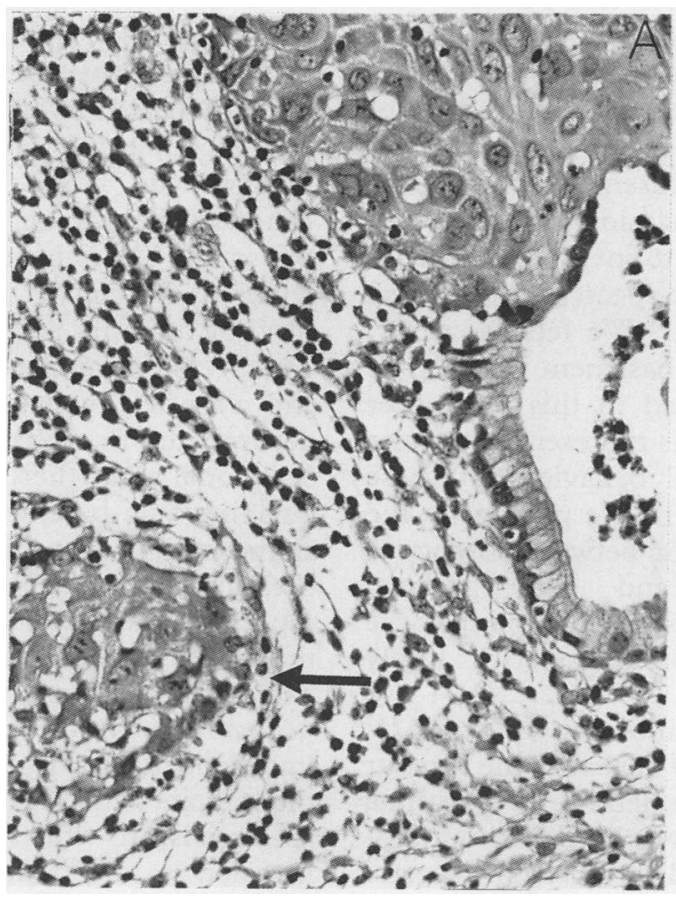

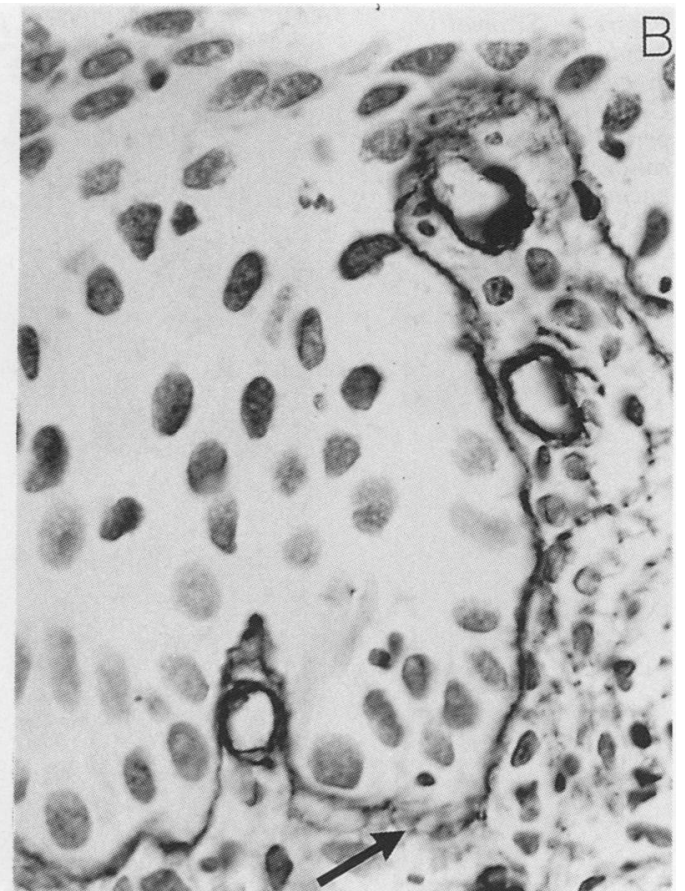

ity for assessing preinvasive and early invasive carcinomas but has also highlighted the diagnostic difficulties which may occur in the identification of early stromal invasion. In one study of microinvasive cervical carcinoma 117 of 232 cases were excluded following histological review because the appropriate criteria for microinvasive disease had not been met; in most cases review suggested that invasion had been overdiagnosed. ${ }^{2}$ The recognition of early invasive disease is nevertheless of clinical importance: first, suspicion of microinvasion in punch biopsy specimens usually requires subsequent cone biopsy of the cervix for accurate staging; second, while the prognosis for early invasive cervical cancer is generally excellent, ${ }^{13}$ occasional cases associated with metastasis have been reported ${ }^{14}$; finally, invasion suggests

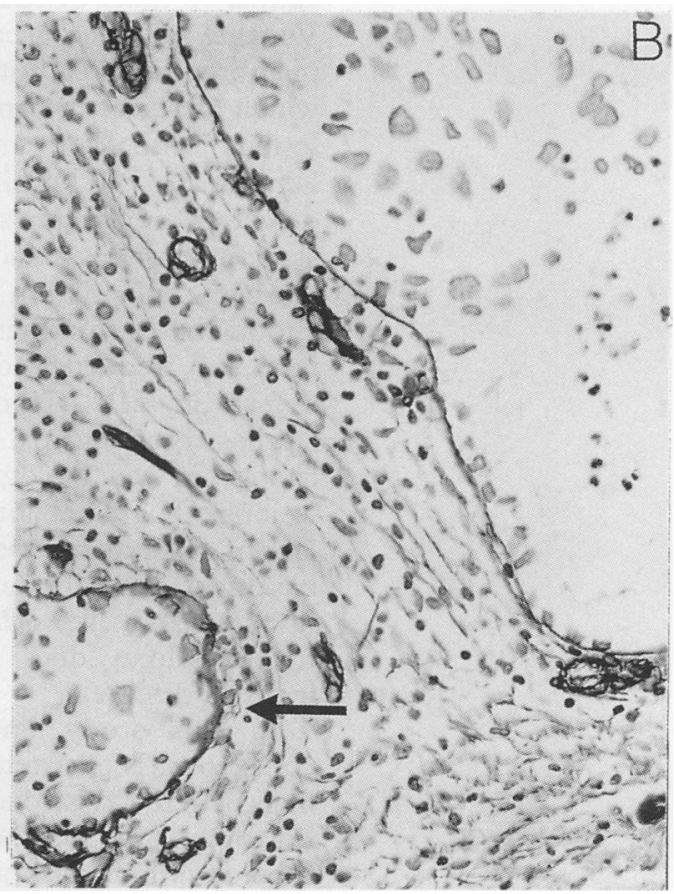


Figure 8 Stromal decidual change in a pregnant patient with CIN III showing pericellular type IV collagen. ( $A)$

haematoxylin and eosin, (B) immunoperoxidase.
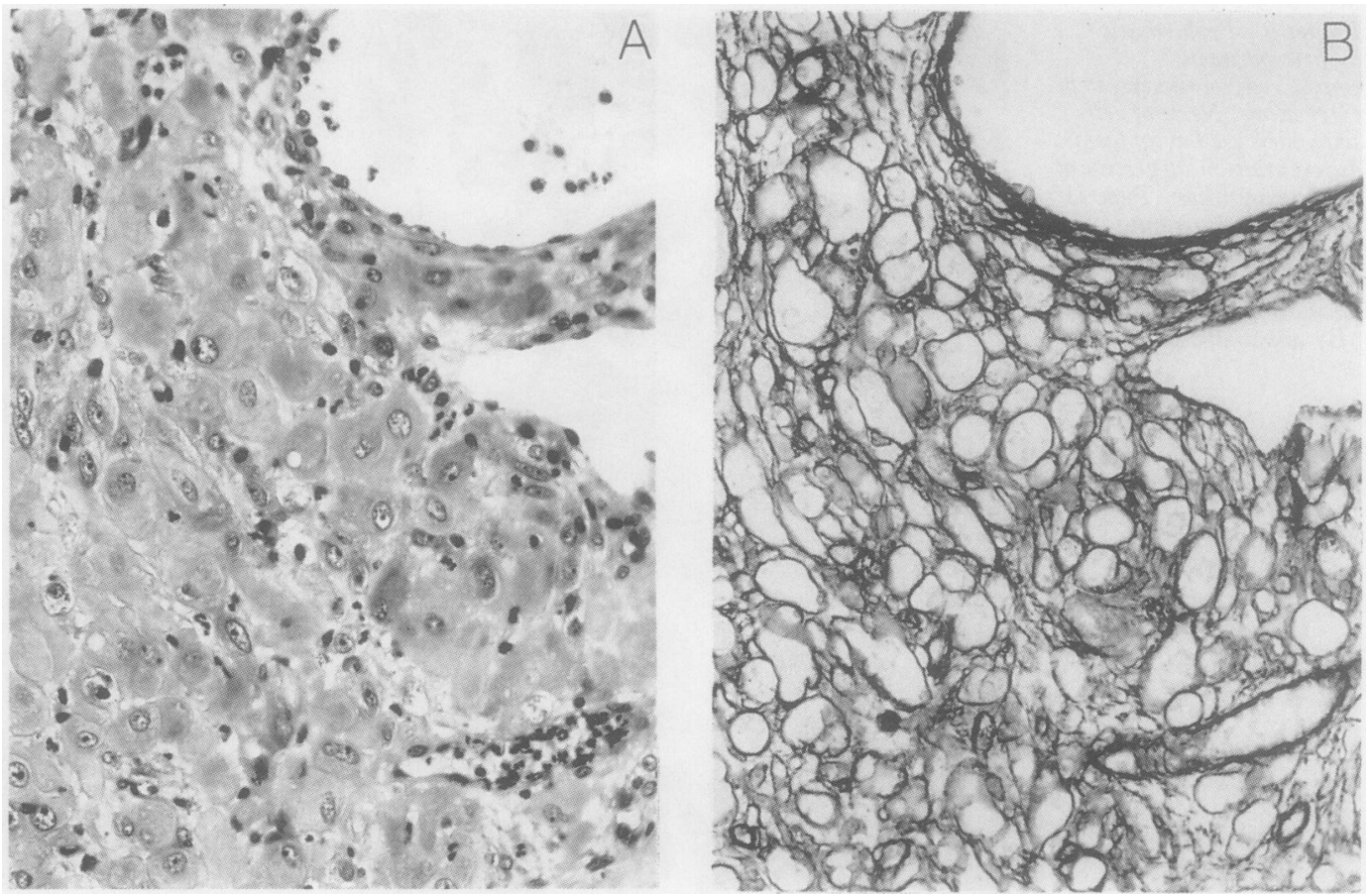

that some change in the balance between the neoplastic process and host responsiveness has taken place.

As breach of the subepithelial basement membrane is generally considered to represent the initial stage of invasion, it has been suggested that immunocytochemical analysis of basement membrane components might help in the assessment of minimal stromal infiltration. Immunocytochemical localisation of type IV collagen has been widely examined and, in general, a continuous intact subepithelial basement membrane has been described in normal tissues as well as in benign and in situ malignant disease. In this study, however, we have found multiple small defects in immunoreactive type IV collagen in more than half the cases of CIN III examined. These findings are similar to those of Richards and Furness ${ }^{7}$ and Vogel et $a l,{ }^{15}$ who used laminin as a basement membrane marker, but differ from other studies which describe an intact pattern in in situ lesions with both type IV collagen and laminin..$^{9116_{17}}$ Focal basement membrane defects in apparent in situ lesions could reflect a stage in the progression to invasive disease as has been suggested in the spectrum of atypical endometrial hyperplasia. ${ }^{18}$ We feel, however, that the frequency of basement membrane defects in CIN III noted in this and other studies argues against this representing a basic change in the biological behaviour of these lesions. Of greater importance perhaps is the lack of absolute correlation between absence of basement membrane and early stromal invasion and, like others, we accept that the former alone does not necessarily imply invasive disease.

In this study immunoreactive type IV collagen was consistently absent at the tumour margin in foci regarded as definite early stromal invasion. This was shown both in buddingtype invasion arising directly from in situ lesions, and around discontinuous, infiltrating cells within the subepithelial tissue. The characteristic morphological changes in such cells seem to be accompanied by a change in biological behaviour, possibly including the ability to degrade basement membrane components. ${ }^{3}$ Such an interaction of malignant cells with extracellular matrix is likely to be critical in tumour behaviour and the ability of tumour cells to adhere to and degrade basement membrane has been shown to correlate with invasive and metastatic potential. ${ }^{19}$

The partial formation of basement membrane around more deeply invasive carcinomas noted in this study is similar to previous reports in tumours of the cervix and other sites. ${ }^{8-11} 1517$ A cyclical process of invasion with initial active destruction of basement membrane followed by a quiescent phase in which membrane components are reformed has been suggested. ${ }^{39}$ We did not observe a correlation between the differentiation of invasive carcinomas and the presence of immunoreactive type IV collagen, as noted by some authors, ${ }^{9} 1517$ although the number of cases examined here was small. The importance of intact or partially retained basement membrane in invasive cervical tumours is uncertain, but a corrrelation between improved short term prognosis and type IV collagen formation has been documented in carcinoma of the bladder. ${ }^{20}$ In occasional areas there was difficulty in distinguishing true basement membrane formation at the margin of tumour invasion from compression of adjacent stromal structures especially vessel walls. This may be resolved by the use of antisera more specific to epithelial basement membrane components. ${ }^{21}$

Several previous studies have suggested that immunocytochemical delination of basement membrane may prove useful in biopsy specimens in which the diagnosis of early invasion is uncertain. No one, however, has 
specifically examined cases in which such a difficulty actually existed. The assessment of such problematic biopsy specimens presented an additional difficulty in that a putative "correct" diagnosis was based on review of routine haematoxylin and eosin stained sections. This assumption clearly imparts a bias to any conclusions and, it could be argued, defeats the purpose of examining such cases by immunocytochemistry. We nevertheless felt that the consistent absence in immunoreactive type IV collagen in both definite and "difficult" cases of early stromal invasion supports the diagnosis in the problematic biopsy specimens. It also implies, however, that immunocytochemical staining of basement membrane added little to the assessment of conventionally prepared material. The mixed pattern of basement membrane staining in problematic specimens which were considered, on review, to show only in situ disease is more difficult to assess: defects in type IV collagen in three of eight cases may indicate underdiagnosis of invasion or could reflect the basement membrane breaks identified in some areas of apparently typical CIN. The presence of an intact, continuous basement membarane in the remaining five biopsy specimens, would, however, support a diagnosis of in situ disease.

In conclusion, early invasive cervical carcinoma is associated with a localised defect in basement membrane type IV collagen immunoreactivity which may reflect an important area of tumour/matrix interaction. In our experience, however, the diagnosis of early stromal invasion can generally be made on morphological changes alone. As defects in basement membrane occur in in situ neoplasia, the value of immunocytochemical localisation of type IV collagen in difficult diagnostic biopsy specimens is also limited.

1 FIGO Cancer Committee. Staging announcement. Gynecol Oncol 1986;25:383-5.
2 Sedlis A, Sall S, Tsukada Y, et al. Microinvasive carcinoma of the uterine cervix: a clinical-pathological study. Am J of the uterine cervix: a clinical-pat

3 Liotta LA. Tumor invasion and metastases: role of the basement membrane. Am J Pathol 1984;117:339-48.

4 Barsky SH, Siegel GP, Jannotta F, Liotta LA. Loss of basement membrane components by invasive tumors but not by their benign counterparts. Lab Invest 1983;49. 140-7.

5 D'Ardenne AJ. Use of basement membrane markers in tumour diagnosis. $J$ Clin Pathol 1989;42:449-57.

6 MacKie RM, Clelland DB, Skerrow CJ. Type IV collagen and laminin staining patterns in benign and malignant and laminin staining patterns in benign and malig

7 Richards CJ, Furness PN. Basement membrane continuity in benign, premalignant and malignant epithelial conditions of the uterine cervix. Histopathology 1990;16: 47-52.

8 Gusterson BA, Warburton MJ, Mitchell D, Kraft N, Hancock WW. Invading squamous cell carcinoma can retain a basal lamina. Lab Invest 1984;51:82-7.

9 Ehrmann RL, Dwyer IM, Yavner D, Hancock WW. An immunoperoxidase study of laminin and type IV collagen distribution in carcinoma of the cervix and vulva. Obstet Gynecol 1988;72:257-62.

10 Pertschuk LP, Boyce JG, Ürcuyo R. An immunofluorescent study of basement membranes in squamous cell carcinoma of the cervix, vagina and vulva. Obstet Gynecol 1977; of the cervis
$49: 417-20$.

11 Frappart L, Berger G, Grimaud JA, et al. Basement membrane of the uterine cervix: immunofluorescence characteristics of the collagen component in normal or atypical epithelium and invasive carcinoma. Gynecol Oncol 1982;13:58-66.

12 Buckley CH, Butler EB, Fox H. Cervical intraepithelial neoplasia. J Clin Pathol 1982;35:1-13.

13 Kolstad PK. Follow-up study of 232 patients with Stage 1al and 411 patients with Stage 1a2 squamous cell carcinoma and 411 patients with Stage 1a2 squamous cell

14 Collins HS, Burke TW, Woodward JE, Spurlock JW, Heller PB. Widespread lymph node metastases in a patient with microinvasive cervical carcinoma. Gynecol Oncol 1989;34:219-21

15 Vogel HP, Mendelsohn G. Laminin immunostaining in hyperplastic, dysplastic, and neoplastic lesions of the endometrium and uterine cervix. Obstet Gynecol 1987;69:794-9.

16 Rubio CA, Biberfeld P, Einhorn N. The immunofluorescence characteristics of the basement membrane in squamous carcinoma of the uterine cervix. Histopathology 1978;2:67-73.

17 Stenback F, Wasenius VM, Risteli J, Risteli L. Basement membranes in progressing intraepithelial cervical neomembranes in progressing intraepithelial cerv

18 Furness PN, Lam EWH. Patterns of basement membrane deposition in benign, premalignant, and malignant endometrium. J Clin Pathol 1987;40:1320-3.

19 Daneker GW, Piazza AJ, Steele GD, Mercurio AM. Interactions of human colorectal carcinoma cells with basement membranes. Arch Surg 1989;124:183-7.

20 Daher N, Abourachid H, Bove N, Petit J, Burtin P. Collagen IV staining pattern in bladder carcinomas: relationship to prognosis. Br J Cancer 1987;55:665-71.

21 Kirkham N, Gibson B, Leigh IM, Price ML. A comparison of antibodies to type VII and type IV collagen: laminin and amnion as epidermal basement membrane markers. $J$ and amnion as epiderm 